\title{
Review of the implementation of plasma ctDNA testing on behalf of IQN Path ASBL: a perspective from an EQA providers' survey
}

\author{
Zandra C. Deans ${ }^{1} \cdot$ Hannah Williams $^{1} \cdot$ Elisabeth M. C. Dequeker $^{2} \cdot$ Cleo Keppens $^{3}$ • \\ Nicola Normanno ${ }^{4} \cdot$ Ed Schuuring ${ }^{5} \cdot$ Simon J. Patton $^{6} \cdot$ Melanie Cheetham ${ }^{6}$. \\ Rachel Butler $^{7}$ - Jacqueline A. Hall ${ }^{8,9}$ • On behalf of IQN Path ASBL
}

Received: 18 April 2017 /Revised: 6 July 2017 / Accepted: 14 August 2017 /Published online: 25 August 2017

(C) The Author(s) 2017. This article is an open access publication

\section{Introduction}

Molecular biomarker analysis for the personalised treatment of non-small cell lung cancer (NSCLC) and colorectal cancer (CRC) is becoming more common, due to the number and availability of molecular targets for predictive biomarker testing increasing [1]. Clinical laboratories must implement accurate test procedures and provide timely and reliable test results, to ensure that appropriate therapies are administered to patients [2]. The challenge for laboratories is to keep pace with molecular biomarker developments while maintaining excellence in service standards.

Plasma circulating tumour DNA (ctDNA) may be found in the blood of cancer patients, alongside a larger fraction of circulating free DNA (cfDNA). Plasma ctDNA testing is becoming more common in the management of cancer patients [3]. It has

This article is part of the Topical Collection on Quality in Pathology

Electronic supplementary material The online version of this article (http://doi.org/10.1007/s00428-017-2222-z) contains supplementary material, which is available to authorized users.

Zandra C. Deans

sandi.deans@ed.ac.uk

1 UK NEQAS for Molecular Genetics, Department of Laboratory Medicine, Royal Infirmary of Edinburgh, Little France Crescent, Edinburgh EH16 4SA, UK

2 Biomedical Quality Assurance Research Unit, Department of Public Health and Primary Care, KU Leuven / University Hospital of Leuven, Leuven, Belgium

3 Biomedical Quality Assurance Research Unit, Department of Public Health and Primary Care, KU Leuven, Leuven, Belgium

4 Cell Biology and Biotherapy Unit, Instituto Nazionale Tumouri "Fondazione Giovanni Pascale", IRCCS, Naples, Italy several advantages: in the absence of suitable or sufficient tissue biopsy, it yields material for molecular analysis, can demonstrate molecular resistance to targeted treatment and is an alternative to invasive tissue sampling [4]. Plasma ctDNA analysis may also prove useful in cases of intra- and inter-tumour heterogeneity [5]. With formal approval from the European Medicine Agency (EMA), several clinical applications for plasma ctDNA testing are now being considered, including the detection of Epidermal Growth Factor Receptor (EGFR) mutations in the plasma of patients with advanced NSCLC [6].

The implementation of new methods such as plasma ctDNA testing can be challenging for diagnostic laboratories. Indeed, it has been shown that inexperience in specialised and complex techniques can compromise the result quality $[2,7]$. To address these issues, four EQA providers came together under the umbrella organisation the International Quality
5 Department of Pathology, University of Groningen, University Medical Center of Groningen, Groningen, The Netherlands

6 European Molecular Genetics Quality Network, Manchester Centre for Genomic Medicine, St Mary's Hospital, Manchester M13 9WL, UK

7 All Wales Genetic Laboratory, Institute of Medical Genetics, University Hospital of Wales, Heath Park, Cardiff CF14 4XW, UK

8 International Quality Network for Pathology (IQN Path ASBL), 3A Sentier de l'Esperance, L-1474 Luxembourg, Luxembourg

9 Division of Cancer, Department of Surgery and Cancer, Imperial College London, London, UK 
Network for Pathology IQN Path (IQN Path): Association Italiana di Oncologia Medica (AIOM), European Molecular Genetics Quality Network (EMQN), European Society of Pathology (ESP) EQA and the United Kingdom National External Quality Assessment Service (UK NEQAS) for Molecular Genetics. Their aim was to survey testing methods currently in use and to pilot an EQA which assessed the standards of plasma ctDNA testing. This article summarises the results of the survey, which evaluated current laboratory practices in this field and which will subsequently inform the design of a pilot EQA scheme for plasma ctDNA testing.

\section{Methods}

An online survey of plasma ctDNA testing practice was designed by the IQN Path collaborative group. The survey was circulated by the four EQA members to their global network of participants, EMQN (1480), UK NEQAS (500), AIOM (47) and ESP testing schemes (568). The survey comprised six sections which included questions about laboratory participation in EQA for solid tumour testing in NSCLC and CRC, current experience and technologies used in plasma ctDNA testing and any analytical limitations of current test methodologies. The survey opened for completion between February 2016 and the middle of March 2016. The responses were analysed to understand current practices in the field of EGFR and RAS mutation testing using ctDNA and will inform design of future pilot EQA scheme.

\section{Results}

Completed surveys were received from 167 laboratories. The submitted data was collated and summarised.

The survey showed that some form of ctDNA plasma testing for EGFR, KRAS and NRAS was used in the majority of responding laboratories $(151 / 167,90 \%)$ but that only $62(37 \%)$ laboratories currently perform diagnostic plasma ctDNA testing (Fig. 1). A further 56 laboratories (34\%) have plasma ctDNA test methodologies in the development phase (Fig. 1). During 2015, 46 diagnostic laboratories tested fewer than 100 samples, while 9 tested more than 101 samples (4 did not respond).

The most frequently used method for plasma ctDNA testing was next-generation sequencing (NGS), used by $27 \%$ of laboratories. The most commonly used testing platform was Ion PGM System ${ }^{\circledR} /$ Thermo Fisher Scientific (Fig. 2a). After NGS, the most frequently used methods were Roche cobas $\AA$, Qiagen therascreen ${ }^{\circledR}$ and ddPCR (Fig. 2a). Of the ddPCR assays, BioRad's QX 200 ddPCR assays were the most commonly used (Fig. 2a).

Of the laboratories using more than one plasma ctDNA testing method, 90 (54\%) employ a single method, 51 (31\%) use

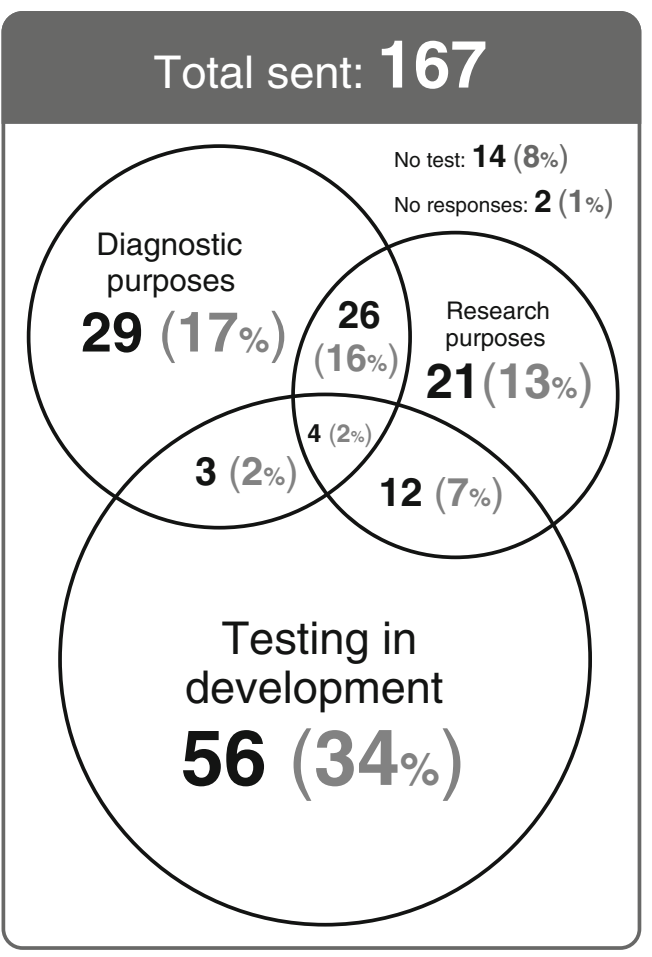

Fig. 1 Summary of the survey results of EGFR, KRAS and NRAS mutation testing in plasma samples. Figures reported are based on the number of laboratories offering testing for either research or diagnostic use or have testing in the development phase. Note: some laboratories use testing for more than one purpose as may be seen by the intersections in the Venn diagram

two and $8(5 \%)$ use three different methods. The remaining 18 did not provide methodology information. Figure $2 b$ illustrates the diversity of methods currently employed in plasma ctDNA testing.

The stated limit of detection (LoD) of all allele frequencies for all laboratories was below $20 \%$. The LoD was $5-20 \%$ in 13 laboratories $(7.8 \%),<5 \%$ in 56 laboratories $(33.5 \%)$ and $<1 \%$ in 62 laboratories $(37.1 \%)$. The remaining laboratories did not provide any LoD data $(21.6 \%)$. Most laboratories performing NGS $(97.8 \%)$ provided an LoD level of $<5 \%$ $(44.2 \%),<1 \%(53.8 \%)$ and $1 \%(<5 \%)$. The other laboratories stated an LoD level of $>20 \%$ or provided no data. For ddPCR, $65.6 \%$ of laboratories gave an $\mathrm{LoD}$ of $<1$ and $21.8 \%$ stated an LoD between 1 and $<5 \%$. A single laboratory stated an LoD of $<10 \%$ and the remaining 3 offered no data.

The EGFR, KRAS and NRAS mutations targeted for analysis in plasma samples were collected. The three most common targets for each gene are outlined in Table 1.

\section{Discussion}

Current practice for plasma ctDNA testing in CRC and NSCLC tumour diagnostic testing was examined by the survey. The laboratories offering plasma ctDNA testing were 
Fig. 2 The breakdown of methodologies used for mutation testing in plasma samples. Values represent the number of laboratories running a specific platform. Some laboratories use more than one testing method. a The current methods used for plasma ctDNA mutation testing by number of laboratories (\% included). b A detailed breakdown of methods specified as "other testing methods"

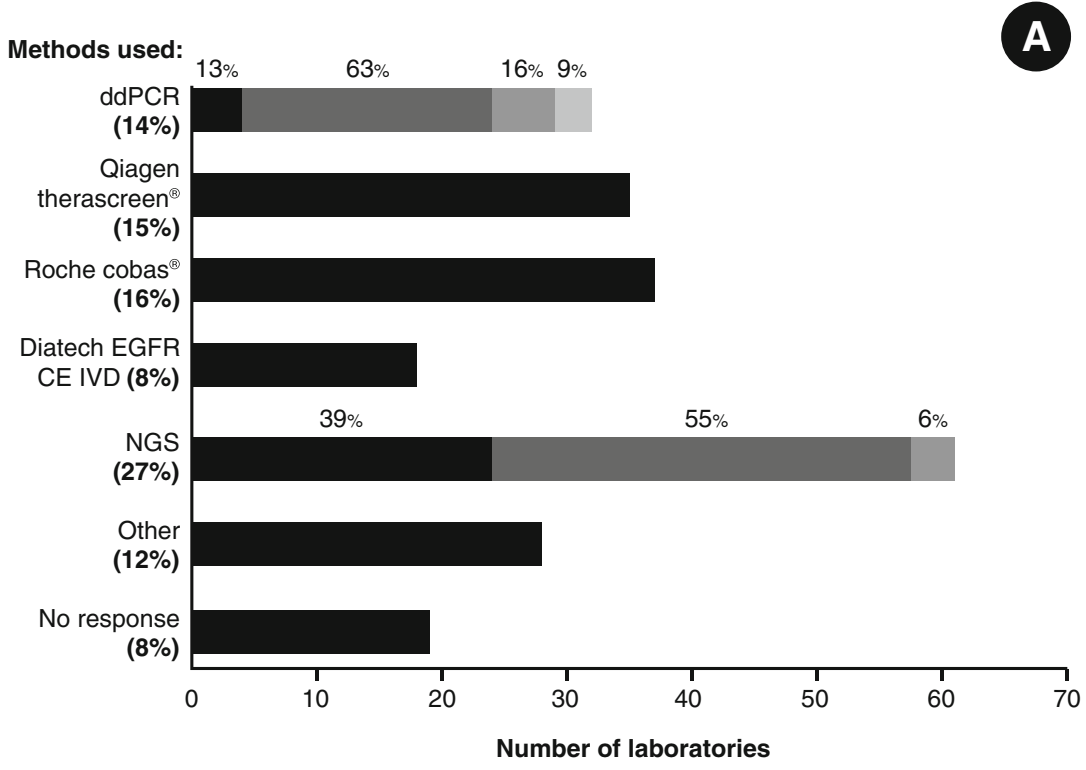

LEGEND

\begin{tabular}{|l|l|}
\hline Digital PCR: & NGS: \\
\hline BEAMing & Other NGS \\
BioRad QX 200 ddPCR & Ion PGM System \\
QuantStudio 3D Thermo Fisher Scientific & Illumina \\
Other ddPCR methods & \\
\hline
\end{tabular}

those which already provide tissue-based molecular pathology services. Most survey participants $(86.8 \%)$ offered diagnostic testing of EGFR, KRAS and NRAS from formalin-fixed paraffin-embedded (FFPE) tissue and participated in EQA for solid tumours. Of these, $78 \%$ participated in EQA assessment for NSCLC and $68 \%$ for CRC, so these laboratories already have experience in molecular technologies and understand how to interpret and report results.

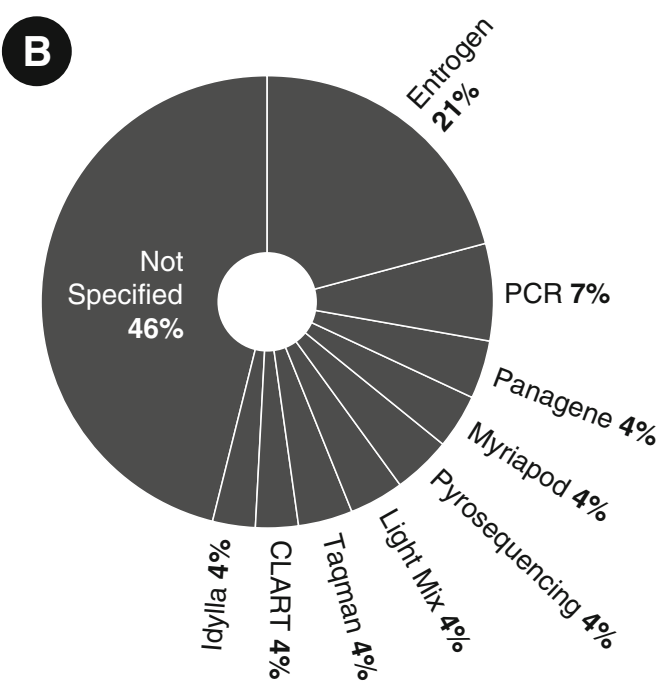

The data suggest that no single, definitive technology for the analysis of plasma ctDNA has yet emerged. The methods currently used are a mixture of commercial and locally developed assays. These assays must be optimised and validated: they must also support adequate test sensitivity and available starting material and must also cover the range of molecular targets that require analysis. 
Table 1 Tabulated frequency of the genes and variants analysed by laboratories for ctDNA testing

\begin{tabular}{|c|c|c|}
\hline Gene & Target & $\begin{array}{l}\text { Number of } \\
\text { laboratory } \\
\text { responses }\end{array}$ \\
\hline \multirow[t]{3}{*}{$E G F R$} & All variants within specified exons & 61 \\
\hline & $\begin{array}{l}\text { p.(G719A), p.(G719C), p.(G719S), } \\
\text { p.(S768I), p.(T790M), p.(L858R), } \\
\text { p.(L861Q), deletions in exon } 19 \\
\text { and insertions in exon } 20\end{array}$ & 28 \\
\hline & $\begin{array}{l}\text { p. }(\mathrm{T} 790 \mathrm{M}), \mathrm{p} .(\mathrm{L} 858 \mathrm{R}) \text { and deletions } \\
\text { in exon } 19\end{array}$ & 27 \\
\hline \multirow[t]{3}{*}{$K R A S$} & All variants within specified exons & 62 \\
\hline & $\begin{array}{l}\text { p.(G12D), p.(G12R), p.(G12A), p.(G12C), } \\
\text { p.(G12S), p.(G12V), p.(G13D) } \\
\text { and p.(Q61H) }\end{array}$ & 13 \\
\hline & $\begin{array}{l}\text { p.(G12D), p.(G12R), p.(G12A), p.(G12C), } \\
\text { p.(G12S), p.(G12V) and p.(G13D) }\end{array}$ & 11 \\
\hline \multirow[t]{3}{*}{ NRAS } & All variants within specified exons & 57 \\
\hline & $\begin{array}{l}\text { p.(G12D), p.(Q61K), p.(Q61R), p.(Q61L) } \\
\text { and p.(Q61H) }\end{array}$ & 10 \\
\hline & $\begin{array}{l}\text { All variants in codons } 12,13,59,117 \text { and } \\
146\end{array}$ & 4 \\
\hline
\end{tabular}

Plasma ctDNA is present at low quantities, mixed within circulating free DNA (cfDNA) in the blood. Therefore, in order to have confidence in the results of plasma ctDNA testing, attention must be paid to the assay sensitivity. However, the optimal sensitivity for ctDNA testing is not yet clear.

An example of a specific clinical application of plasma ctDNA is the phase IV EGFR tyrosine kinase inhibitor Gefitinib 'Follow Up Measure' trial that facilitated the approval of plasma ctDNA testing for EGFR in NSCLC patients (6). The trial showed that although the Qiagen therascreen ${ }^{\circledR}$ kit had a low sensitivity (65.7\%), it had a good correlation with the response of patients to first-line treatment with Gefitinib [4].

In the future, more sensitive techniques are likely to detect more patients with $E G F R$ mutations and may also identify $E G F R$ variants in patients with heterogeneous expression [8].

The use of highly sensitive ctDNA testing methods has permitted new insights into heterogeneity, e.g. p.T790M mutations in the ctDNA of patients with a tumour mass that tested negative for the resistance mutation [9]. Patients with positive plasma ctDNA tests and negative tissue results had shorter progression-free survival compared to patients with $E G F R$ p.T790M detected in both their tumour tissue and plasma ctDNA [9]. In the future, accurate measurements of the ratio of resistant EGFR mutations to sensitising mutations might help select patients who are more likely to benefit from treatment with drugs targeting p.T790M [10].

Similarly, NGS panels may be used to determine the relative abundance of a tumour variant to support individually tailored therapy. Where NGS is used, a broad range of molecular targets may be detected simultaneously; however, this may be at the cost of lower test LoD. Non-NGS-based methods may provide greater sensitivity but have the limitation of assaying fewer molecular targets.

There is a significant interest in the development of plasma ctDNA services. However, despite the 2014 EMA approval for plasma ctDNA biopsies which determine the suitability of first-line treatment of NSCLC with Gefitinib, few laboratories currently deliver NSCLC or CRC clinical diagnostic services [11]. For plasma ctDNA testing to become integrated into routine practice, those offering clinical services must be educated on its applications. However, until local services can validate and embed testing in patient pathways, laboratory and clinical uptake of plasma ctDNA may be hindered.

Current clinical applications for ctDNA are largely confined to NSCLC and CRC, although there is potential for its use in many other areas of oncology. Laboratories must provide high-quality testing services in which clinical teams and patients have confidence. The delivery of National and International EQA schemes is essential to maintain quality through the standardisation of sample logistics, molecular assays and result interpretation, as well as playing an important role in supporting education [4].

As many laboratories plan to implement the testing of plasma ctDNA, it is clear that support from well-designed EQA schemes is needed. Without this support, laboratories may be slower to offer plasma ctDNA clinical services or may encounter issues. Surveying current practices and collecting data to inform EQA design is a task that may be harmonised between several EQA providers, all with the aim of increasing efficiencies and supporting best practice standards in quality assessment [12].

Acknowledgements We would like to thank the sponsors who supported the plasma ctDNA pilot of IQN Path, the initial step of which is presented here by the survey results. Sponsors include Amgen, AstraZeneca, Merck KGA, Sysmex Inostics, Boehringer Ingelheim, Roche and Thermo Fisher Scientific.

Funding IQN Path provided administrative support for the project.

Compliance with ethical standards This research does not contain any studies involving human participants or animals performed by any of the authors. Human subjects were not used; therefore, formal consent was not required.

Conflict of interest Zandra C Deans, Hannah Williams, Cleo Keppens, Simon J Patton, Melanie Cheetham and Rachel Butler declare that they have no conflict of interest. Elisabeth MC Dequeker receives grants from Roche and Pfizer. Nicola Normanno receives grants and personal fees from AstraZeneca, Qiagen, Roche, Merck Serono, Sysmex. Ed Schuuring is on the Advisory Board and receives personal fees and travel expenses from Novartis, Pfizer and AstraZeneca and receives travel fees only for participation in an Advisory Board for Amgen. He is also on an advisory board for QCMD and receives a grant for laboratory costs. Jacqueline Hall owns stock in Vivactiv Ltd. 
Open Access This article is distributed under the terms of the Creative Commons Attribution 4.0 International License (http:// creativecommons.org/licenses/by/4.0/), which permits unrestricted use, distribution, and reproduction in any medium, provided you give appropriate credit to the original author(s) and the source, provide a link to the Creative Commons license, and indicate if changes were made.

\section{References}

1. Cree IA, Deans Z, Ligtenberg MJ, Normanno N, Edsjo A, Rouleau E, Sole F, Thunnissen E, Timens W, Schuuring E, Dequeker E, Murray S, Dietel M, Groenen P, Van Krieken JH, European Society of Pathology Task Force on Quality Assurance in Molecular Pathology, Royal College of Pathologists (2014) Guidance for laboratories performing molecular pathology for cancer patients. J Clin Pathol 67:923-931. https://doi.org/10.1136/ jclinpath-2014-202404

2. Dubbink HJ, Deans ZC, Tops BB, van Kemenade FJ, Koljenovic S, van Krieken HJ, Blokx WA, Dinjens WN, Groenen PJ (2014) Next generation diagnostic molecular pathology: critical appraisal of quality assurance in Europe. Mol Oncol 8:830-839. https://doi. org/10.1016/j.molonc.2014.03.00410.1016/j.molonc.2014.03.004

3. Rolfo C, Castiglia M, Hong D, Alessandro R, Mertens I, Baggerman G, Zwaenepoel K, Gil-Bazo I, Passiglia F, Carreca AP, Taverna S, Vento R, Peeters M, Russo A, Pauwels P (2014) Liquid biopsies in lung cancer: the new ambrosia of researchers. Biochim Piophys Acta 1846:539-546. https://doi.org/10.1016/j. bbcan.2014.10.001

4. Douillard JY, Ostoros G, Cobo M, Ciuleanu T, Cole R, McWalter G, Walker J, Dearden S, Webster A, Milenkova T, McCormack R (2014) Gefitinib treatment in EGFR mutated caucasian NSCLC: circulating-free tumour DNA as a surrogate for determination of EGFR status. J Thorac Oncol 9:1345-1353. https://doi.org/10. 1097/JTO.0000000000000263

5. Normanno N, Rachiglio AM, Roma C, Fenizia F, Esposito C, Pasquale R, La Porta ML, Iannaccone A, Micheli F, Santangelo M, Bergantino F, Costantini S, De Luca A (2013) Molecular diagnostics and personalised medicine in oncology: challenges and opportunities. J Cell Biochem 114:514-524. https://doi.org/ $10.1002 / j \mathrm{jcb} .24401$

6. Douillard JY, Ostoros G, Cobo M, Ciuleanu T, McCormack R, Webster A, Milenkova T (2014) First-line gefitinib in Caucasian EGFR mutation-positive NSCLC patients: a phase-IV, open-label, single-arm study. Br J Cancer 110:55-62. https://doi.org/10.1038/ bjc. 2013.721

7. Tack V, Ligtenberg MJ, Tembuyser L, Normanno N, Vander Borght S, Han van Krieken J, Dequeker EM (2015) External quality assessment unravels interlaboratory differences in quality of RAS testing for anti-EGFR therapy in colorectal cancer. Oncologist 20: 257-262. https://doi.org/10.1634/theoncologist.2014-0382

8. Rachiglio AM, Abate RE, Sacco A, Pasquale R, Fenizia F, Lambiase M, Morabito A, Montanino A, Rocco G, Romano C, Nappi A, Laffaioli RV, Tatangelo F, Botti G, Ciardiello F, Maiello MR, De Luca A, Normanno N (2016) Limits and potential of targeted sequencing analysis of liquid biopsy in patients with lung and colon carcinoma. Oncotarget 7:66595-66605. 10.18632/ oncotarget.10704

9. Oxnard GR, Thress KS, Alden RS, Lawrance R, Paweletz CP, Cantarini M, Yang JC, Barrett JC, Jänne PA (2016) Association between plasma genotyping and outcomes of treatment with osimertinib (AZD9291) in advanced non-small-cell lung cancer. J Clin Oncol 34:3375-3382. https://doi.org/10.1200/JCO.2016.66. 7162

10. Normanno N, Maiello MR, Chicchinelli N, Iannaccone A, Esposito C, De Cecio R, D'alessio A, De Luca A (2017) Targeting the EGFR T790M mutation in non-small-cell lung cancer. Expert Opin Ther Targets 21:159-165. https://doi.org/10.1080/14728222.2017. 1272582

11. Annex 1. Summary of product characteristics. European Medicines Agency http://www.ema.europa.eu/docs/en_GB/document_library/ EPAR_-_Product_Information/human/001016/WC500036358.pdf. (last accessed on 27/12/2016)

12. van Krieken JH, Normanno N, Blackhall F et al (2013) Guideline on the requirements of external quality assessment programs in molecular pathology. Virchows Arch 462:27-37. https://doi.org/ 10.1007/s00428-012-1354-4 